\title{
HIV/AIDS, Trypanosomiasis and Malaria Interaction, a Case Report
}

\author{
Article by Mudji E'kitiak \\ M.Sc., Clinical Research, Texila American University, Congo \\ Email: mudjimedecin@yahoo.fr
}

\begin{abstract}
A number of infectious diseases remain neglected, such as the sleeping sickness and often occur in individuals who are additionally affected by HIV/AIDS and malaria, making the problem even more serious. In this article we will be discussing the clinical interactions between HIV/AIDS, malaria and trypanosomiasis infection.

The discussed patient is a 34 year old female who presented with an intermittent fever lasting approximately three months and behavioural problems persisting for one week. The patient reported unprotected sex with multiple sexual partners. She was diagnosed with HIV (WHO stage III), human African trypanosomiasis stage II and malaria with high parasitemia. The patient receives treatment for malaria and trypanosomasis but died of neurological causes.

Discussion: There is a scarcity of data regarding the coexistence of HIV/AIDS, trypanosomasis, and malaria in a specific patient. This creates a complicated situation for diagnosis and treatment.

Conclusion: Co-infections through various infectious agents are common in sub-Saharan African creating important diagnostic, therapeutic, and prognostic implications.
\end{abstract}

\section{Introduction}

Infectious diseases remain a major health and socioeconomic problem in many low-income countries, particularly in sub-Saharan Africa (1). For many years, the three most devastating infectious diseases: HIV/AIDS, malaria, and tuberculosis (TB) have received most of the world's attention (1). However, in rural and impoverished urban areas, a number of infectious diseases, such as sleeping sickness remain neglected (1) and often occur in individuals who are additionally affected by HIV/AIDS and malaria. These co-infections are the rule rather than the exception in many geographical areas (1). For the past three decades, the Democratic Republic of the Congo (DRC) has been reporting the world's highest number of human African trypanosomiasis (HAT) cases (2). In 2012, the DRC continued to bear the heaviest burden of trypanosoma brucei gambiense HAT, accounting for $84 \%$ of all cases reported in the African continent (2). Even with the recent decrease in malaria disease, it remains a significant health problem (3). Sub-Saharan Africa, the area hardest hit by the HIV/AIDS pandemic worldwide, is home to nearly 25 million people living with HIV/AIDS (4). In this article we will discuss the clinical interactions between HIV/AIDS, malaria and trypanosomiasis infection.

\section{Patient presentation}

The patient, a 34 year old female, was brought in for consultation with the following symptoms:

An intermittent fever over approximately three months and behavioural problems lasting for a week. Family history is unremarkable and her parents are alive and reportedly in good health. She is the second child born to her parents who have a total of five children, two girls and three boys. She is a single mother of two children from different fathers; the children are healthy and her youngest is four years old. The patient divorced the father of her first child and is currently living with the father of her second child. She sells small items in a shop in Kinshasa the capital of DRC. There is no known major surgical or medical history. Date of last menstrual cycle is unknown 
South American Journal of Public Health

Special Edition May 2016

\section{History of present illness}

About two months prior to the initial consultation (April 2011) the patient had episodes of fever and weight loss. She consulted a small health centre in Kinshasa where she received unknown treatment. The fever persisted and the patient's behaviour changed so the family decided to travel over $500 \mathrm{~km}$ to the Vanga Evangelical Hospital. The family reported logorrhoea, persistent fever, refusal to eat for a week and loss of an unknown amount of weight. The patient reported unprotected sex with multiple sexual partners.

\section{Physical examination}

Vital Signs: Blood pressure: 100/60 mmHg, Heart rate: 106 beat per minute, Respiratory rate: 30 per minute, Temperature: $39^{\circ} \mathrm{C}$, Weight: $36 \mathrm{~kg}$ and Height: $148 \mathrm{Cm}$.

Normal conjunctiva and anicteric bulbar conjunctiva. No palpable lymph nodes. Her mouth was moist with white coated tongue due to the thrush. Cardiopulmonary examination was normal. The abdomen was not distended and soft. Tenting of the skin from dehydration was noted on the abdomen. Neurological examination showed logorrhoea and disorientation to both time and place. Cranial nerves were tested and proved normal and symmetric. The sensation exam, motor exam, and reflexes were normal. She had several excoriations on her legs and arms.

\section{Para-clinical examinations}

White Blood Cell count: 4,600/mm3, WBC differential: Neutrophils 82\% Lymphocytes 18\%; Thick smear for malaria highly positive; Haemoglobin: 10g\%; CATT: positive, CATT diluted to 1/16: positive; HIV positive; Lumbar puncture: clear appearance, white blood cells: 150 cells/field. CSF trypanosome positive; Cryptococcus negative; Sedimentation rate: $120 \mathrm{~mm} /$ hour

\section{Clinical assessment}

The main problem was the combination of HIV infection (WHO stage III) along with oral candidiasis, human African trypanosomiasis (stage II), and malaria with high parasitemia

\section{Management}

Clinical Treatment

- Cotrimoxazole 960mg for two weeks.

- Antiretroviral medication was under consideration.

- Fluconazole $300 \mathrm{mg} / \mathrm{day}$.

- Haloperidol $10 \mathrm{mg} /$ day.

- Quinine (1g in 500ml 5\% glucose solution over 4 hours continued with $450 \mathrm{mg}$ quinine every 8 hours for 7 days)

- NECT was given at a rate of

- DFMO 2x $36 \mathrm{ml} /$ day/7days and

- Nifurtimox 3x1.5 g/10 days.

The fever resolved but the abnormal behaviour continued and the patient refused to eat and drink.

Lumbar puncture was performed at the end of the trypanosomiasis treatment with the following result: White Blood Cell: 8 elements. The CSF for trypanosomes and the thick blood smear for malaria were negative but the HIV status remained positive.

Ultimately the patient became comatose and died during the third week of hospitalization.

\section{Discussion}

Interaction hiv/ trypanosomiasis/malaria.

Co-infections with malaria and trypanosome were studied in detail by Irene Kuepfer (5). They showed that in a patient population from Tanzania the majority (80\%) of the patients were malaria-positive upon admission. These patients had a higher rate of pruritus, sleeping 
disorders, anorexia and abnormal behaviour. However, there was insufficient evidence for profound differences in malaria-positive and malaria-negative subjects, which may have been due to asymptomatic carries.

The HIV status of the patient did not change the clinical presentation or the treatment outcomes of $T$.b. rhodesiense HAT (5). Patients in central and western Africa have shown a higher affinity to HIV type 2 and when treated appeared to recover from HAT but later succumb to full-blown AIDS (6). In another study conducted in Congo HIV had no significant impact on the incidence of trypanosomiasis (T.b. gambiense) but might cause a higher risk of treatment failure of trypanosomiasis (7). The same results were observed in the Ivory Coast. (8). In Congo a recent study showed that Gambian trypanosome infection decreases the sensitivity of HIV antibody tests (9).

Regarding the cause of death, the patient had HIV and thrush but without neurological abnormality such as a tumour or HIV encephalitis (10). The decrease count of white cells shows that, NECT treatment was efficient. The cause of the death remains unknown: It is possible, that the treatment begun in a to advanced stage of the disease and the treatment could no longer heal the patient. Another possibility is a treatment related encephalopathy reaction, which was often reported in association with Melarsoprol, but also with NECT (11). Of 18 patients treated with melarsoprol for second stage HAT in a rural hospital in the DRC, 4/18 tested HIV positive (11). All 14 HIV seronegative patients had a favourable outcome of treatment and left the hospital in good health, whereas the four HIV co-infected patients had an unfavourable outcome (11). Three died during treatment probably because of an encephalopathy syndrome and one left the hospital in unchanged clinical condition, despite treatment of HAT (11).

In trypanosome brucei gambiense patients, heart involvement as shown by ECG alterations where frequently observed (12). ECG findings were characterized predominantly as low voltage and repolarization changes, and to a lesser extent as PR depression and QTC prolongation (12). QTc prolongation may have clinical relevance when new drugs with the potential for QTc interaction are used (12). In our patient an ECG was not done due to the lack of cardiac symptoms and arrythmnias leading to the coma could not be ruled out.

\section{Conclusion}

Co-infection of various infection agents is common in sub-Saharan African which has important diagnostic, therapeutic, and prognostic implications.

Acknowledgement I would like to acknowledge Dr Johannes BLUM, Dr timothy RICE, Dr Paul TUTTLE, Philipp SAUER for their support while writing this article.

\section{References}

[1.] Afro.who.int. Overview: HIV/AIDS - WHO | Regional Office for Africa [Internet]. 2015 [cited 10 August 2015]. Available from: http://www.afro.who.int/en/clustersa-programmes/dpc/acquired-immune-deficiency-syndrome/overview.html

[2.] Blum J, Burri C, Hatz C, Kazumba L, Mangoni P, Zellweger M. Sleeping hearts: the role of the heart in sleeping sickness (human African trypanosomiasis). Tropical Medicine \& International Health. 2007;12(12):1422-1432.

[3.] Blum J, Nkunku S, Burri C. Clinical description of encephalopathic syndromes and risk factors for their occurrence and outcome during melarsoprol treatment of human African trypanosomiasis. Trop Med Int Health. 2001;6(5):390-400.

[4.] Boraschi D, Abebe Alemayehu M, Aseffa A, Chiodi F, Chisi J, Del Prete G et al. Immunity against HIV/AIDS, Malaria, and Tuberculosis during Co-Infections with Neglected Infectious Diseases: Recommendations for the European Union Research Priorities. PLoS Neglected Tropical Diseases. 2008;2(6):e255.

[5.] Galactionova K, Tediosi F, de Savigny D, Smith T, Tanner M. Effective Coverage and Systems Effectiveness for Malaria Case Management in Sub-Saharan African Countries. PLOS ONE. 2015;10(5):e0127818.

[6.] Kuepfer I, Hary E, Allan M, Edielu A, Burri C, Blum J. Clinical Presentation of T. b. rhodesiense 
South American Journal of Public Health

Special Edition May 2016

Sleeping Sickness in Second Stage Patients from Tanzania and Uganda. PLoS Negl Trop Dis. 2011;5(3):e968.

[7.] Lejon V, Ngoyi D, Ilunga M, Beelaert G, Maes I, Buscher P et al. Low Specificities of HIV Diagnostic Tests Caused by Trypanosoma brucei gambiense Sleeping Sickness. Journal of Clinical Microbiology. 2010;48(8):2836-2839.

[8.] Lumbala C, Simarro P, Cecchi G, Paone M, Franco J, Kande Betu Ku Mesu V et al. Human African trypanosomiasis in the Democratic Republic of the Congo: disease distribution and risk. International Journal of Health Geographics. 2015;14(1).

[9.] Matete G, Kajejo O. Human African Trypanosomiasis And Human Immunodeficiency Virus Co-Infection In Western Kenya. E Af Med Jrnl. 2005;82 (1).

[10.] Meda H, Doua F, Laveissière C, Miezan T, Gaens E, Brattegaard K et al. Human immunodeficiency virus infection and human African trypanosomiasis: a case-control study in Côte d'Ivoire. Transactions of the Royal Society of Tropical Medicine and Hygiene. 1995;89(6):639-643.

[11.] Pepin J, Ethier L, Kazadi C, Milord F, Ryder R .The impact of human immunodeficiency virus infection on the epidemiology and treatment ofTrypanosoma brucei gambiense sleeping sickness in Nioki, Zaire. Am J Trop Med Hyg 47.1992;47(7)

[12.] Tack D, Holman R, Folkema A, Mehal J, Blanton J, Sejvar J. Trends in Encephalitis-Associated Deaths in the United States, 1999-2008. Neuroepidemiology. 2014;43(1):1-8.11. 